Suchodolska Magdalena, Świerczyńska Blanka, Undziakiewicz Adrian, Smoluchowski Krzysztof, Sekuła Michal. Risk factors for gallbladder cancer. Journal of Education, Health and Sport. 2020;10(9):487-493. eISSN 2391-8306. DOI http://dx.doi.org/10.12775/JEHS.2020.10.09.058

https://apcz.umk.pl/czasopisma/index.php/JEHS/article/view/JEHS.2020.10.09.058

https://zenodo.org/record/4042153

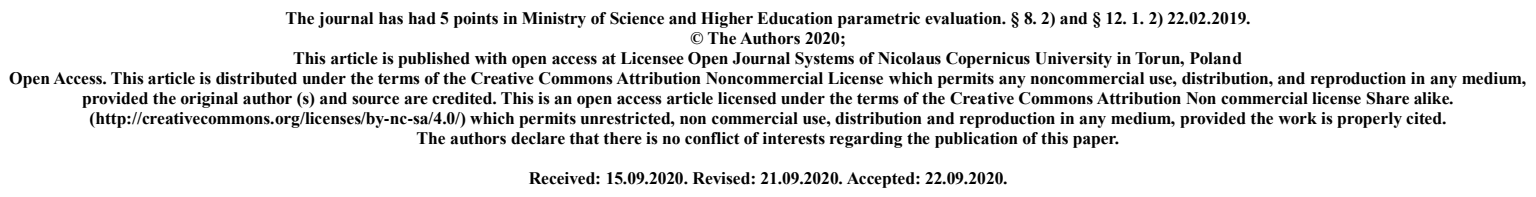

\title{
Risk factors for gallbladder cancer
}

\section{Magdalena Suchodolska ${ }^{1}$, Blanka Świerczyńska ${ }^{1}$, Adrian Undziakiewicz ${ }^{1}$, Krzysztof Smoluchowski ${ }^{1}$, Michał Sekuła ${ }^{1}$}

${ }^{1}$ Student Research Group of Oncological Surgery, Medical University of Lublin, Poland

Magdalena Suchodolska, https://orcid.org/0000-0002-1995-5136;

magda.suchodolska.1998@ interia.eu (corresponding author)

Blanka Świerczyńska, https://orcid.org/0000-0001-8782-8625;

swierczynska.blanka@gmail.com

Adrian Undziakiewicz, https://orcid.org/0000-0002-1191-1366;

adrian.undziakiewicz@gmail.com

Krzysztof Smoluchowski, https://orcid.org/0000-0001-9237-3346;

ksmoluchowski@gmail.com

Michał Sekuła, https://orcid.org/0000-0001-8378-9964; michalsekula061996@ gmail.com 


\section{Abstract \\ Introduction and purpose:}

Gallbladder cancer is the most common neoplasm of the bile duct. Early GBC is usually asymptomatic, but is prone to early metastases. This means that most GBC cases are diagnosed very late, and this contributes to the poor prognosis of this disease. Less than $20 \%$ of GBC cases qualify for a potentially curable surgical resection at the moment of diagnosis. The article is an overview of available literature describing current epidemiology and etiological risk factors associated with the development of gallbladder cancer (GBC). We performed a review of the articles on the etiology and GBC carcinomatosis.

\section{Brief description of the state of knowledge:}

We divided risk factors of GBC into independent and dependent. The independent risk factors are: age, sex, cholelithiasis, porcelain gallbladder, gallbladder polyp, anomalous junction of the pancreaticobiliary ductal system, high BMI and obesity, and Helicobacter pylori. On the other hand, the dependent risk factors are: age of first pregnancy and number of pregnancies, xanthogranulomatous cholecystitis, lipid peroxidation products, and aflatoxins. Gallbladder cancer appears to be a multifactorial disease, but the main risk factors are: cholelithiasis and porcelain gallbladder developing from chronic cholecystitis.

\section{Conclusions:}

For understanding the etiopathology of GBC more studies have to be conducted, what may allow to establish reliable biomarkers and invent new preventive strategies and more effective treatment.

Keywords: gallbladder cancer, cholelithiasis, risk factors.

\section{Introduction}

The gallbladder (GB) is responsible for the storage and concentration of the bile produced by hepatocytes in the liver. It is located in the right hypochondrium, below the right lobe of the liver. Anatomically, GB is divided into three sections: the fundus, body, and neck that becomes continuous with the cystic duct. The cystic duct unites with the common hepatic duct to become the common bile duct, which is later joined by the pancreatic duct to form the ampulla of Vater. This is the way of bile excretion from the liver to duodenum.

Gallbladder cancer (GBC) develops from the gallbladder mucosa and its most common histological type is adenocarcinoma. It was first described in 1777 by Stoll [1]. Gallbladder cancer is the most common neoplasm of the bile duct [2]. The overall incidence of gallbladder cancer worldwide is low. According to the International Agency for Research on Cancer (IARC), Globocan 2018 database GBC took 20th place in the world in terms of the number of new cases of malignant neoplasms; 219,420 cases were diagnosed at that time [3]. Accurate estimate of the prevalence of gallbladder cancer worldwide is difficult to obtain due to the difficulties in detecting and diagnosing gallbladder cancer, especially in less developed countries. Early GBC is usually asymptomatic, but is prone to early metastases. This means that most GBC cases are diagnosed very late, and this contributes to the poor prognosis of this disease. Less than $20 \%$ of GBC cases qualify for a potentially curable surgical resection at the moment of diagnosis. 
This article provides an overview of the current epidemiology and in this article we have reviewed the etiological risk factors associated with gallbladder cancer development.

\section{Epidemiology}

There is a significant geographic differentiation in terms of GBC incidence. The highest incidence is observed in Chile (27/100,000) and India (21.5/100,000). Moreover, it is noticeable that the much higher incidence of GBC is observed in ethnic groups in Asia or Native Americans compared to non-Native Americans (3.3/100,000 vs. 0.4-1.5/100,000) [4], [5]. The lowest incidence rates are observed in highly developed countries such as the USA, Australia, Great Britain and Canada. In Europe, the highest incidence was found in the countries of Eastern Europe: Poland and the Czech Republic. In 2018, the International Agency for Research on Cancer (IARC) Globocan 2018 database estimated gallbladder cancer to constitute $1.7 \%$ of all cancer deaths.

\section{Risk factors for gallbladder cancer}

The exact etiology of GBC is not entirely clear. In our work, we divided risk factors into independent and dependent.

\subsection{Independent etiological factors}

Age

$\mathrm{GBC}$ is a result of the progression of dysplasia to pre-invasive and invasive cancer. This process takes about 15 years [1]. Severe dysplasia and carcinoma in situ have been found in more than $90 \%$ of gallbladders that contain GBC [2]. According to data, the average age of patients with early cancer was 56.8 years, advanced carcinoma 62.9 years and metastasis of GBC 63.1 years [3]. The progression from intraepithelial lesions to carcinoma takes time. Most cases of GBC occur over the age of 50 (95\% of cases in both sexes), with over $50 \%$ of cases occurring over the age of 70 [4].

\section{Sex}

GCS affects women 2 to 6 times more commonly than men. The estrogen causes increased cholesterol concentration in bile and predisposes to development of cholelithiasis and indirectly GBC [5]. The role of female hormones in the development of gallbladder cancer is still uncertain and requires further research.

\section{Cholelithiasis}

According to clinical trials, cholelithiasis remains one of the major risk factors for the development of GBC. However there is no experimental data to support them [6], [7]. The exact mechanism that predisposes people with cholelithiasis to develop gallbladder cancer is not fully understood. Probably the presence of stones in the gallbladder can cause chronic inflammation, which promotes the development of dysplasia and even adenocarcinoma. However, it is worth emphasising that only $0.3 \%$ to $3 \%$ of patients with cholelithiasis develop GBC. 
On the other hand, about $20 \%$ of patients with GBC have not been diagnosed with cholelithiasis [8]. Studies suggest that the risk of gallbladder cancer is 10 times higher in patients with gallstones $>3 \mathrm{~cm}$ in diameter compared to patients with stones $<1 \mathrm{~cm}$ [9].

\section{Porcelain gallbladder and gallbladder polyp}

Porcelain gallbladder and gallbladder polyps are other risk factors for GCB. Porcelain gallbladder is defined as calcification of gallbladder wall and is believed to be an end stage of chronic cholecystitis. This disease coexists with GBC in $12.5 \%$ to $62 \%$ of patients [10]. Selective mucosal calcification of gallbladder wall is more dangerous compared to complete intramural calcification [11]. Polyps $>10 \mathrm{~mm}$ in diameter should be removed, in symptomatic patients with polyps $<10 \mathrm{~mm}$ in diameter cholecystectomy is recommended [12].

\section{Anomalous junction of the pancreaticobiliary ductal system}

An anomalous junction of the pancreaticobiliary ductal system (AJPBDS) is the reason of the regurgitation of pancreatic juice into the gallbladder and cholestasis, which in turn leads to inflammation of the gallbladder mucosa, dysplasia and, consequently, the development of GBC. In one retrospective study, AJPBDS occurred in $17.8 \%$ of patients with GBC [13].

\section{Body Mass Index and Obesity}

Body Mass Index (BMI) is a measure of body fat based on height and weight that applies to adult men and women. It is a person's weight in kilograms divided by the square of height in meters. High BMI is significantly linked with higher risk of biliary tract disease (both of biliary tract cancer and non-cancer biliary tract disease), the association for BMI is more expressed among women than men [14]. Risk of biliary tract cancer is 1.4 times higher in obese patients compared to patients with normal BMI 19. The results of meta-analysis showed that the relative risk (RR) is 1.11 per $5 \mathrm{~kg} / \mathrm{m} 2$ increase in BMI in men and women, respectively 19 . The exact mechanisms linking obesity and GBC are not entirely clear. There are several potential possibilities. Obesity is associated with chronic low-grade inflammation, which is more expressed in the visceral than subcutaneous adipose tissue. There are multiple possible mechanisms responsible for initiation and development of inflammation of adipose tissue in obese patients. Adipose tissue is infiltrated by M1-macrophages, which secrete inflammatory factors such as interleukin-6 (IL 6) and tumour necrosis factor- $\alpha$ (TNF $\alpha$ ). Chronic low-grade inflammation associated with obesity may contribute to biliary tissue damage, dysplasia and cancer development [15]. According to the current knowledge, controlling and maintaining a healthy body weight can help minimize the risk of gallbladder cancer [16].

\section{Helicobacter pylori}

H. pylori, previously known as Campylobacter pylori, is a Gram-negative, microaerophilic bacterium, that colonizes the gastrointestinal tract. H. pylori is indicated as cause of chronic cholecystitis, primary sclerosing cholangitis, and primary hepatic carcinomas. Chronic infection of the gallbladder with Helicobacter pylori and has been suggested as a risk factor for GBC [17]. 
Chronic inflammation increases the frequency of expression of cancers [18]. Murphy et al. in their study emphasized that the H. pylori antigen predominance was present in $100 \%$ of the gallbladder cancer cases and concluded that $H$. pylori proteins was accompanied with the risk of biliary tract cancers [19].

\subsection{Dependent etiological risk factors}

\section{Age of first pregnancy and number of pregnancies}

The age at first pregnancy was inversely and significantly associated with the biliary tract cancer [20]. According to a study by Clara J. Moerman, women who are pregnant for the first time at the age of 23 years or younger are at a 2 times higher risk of bile duct cancer compared to women who were pregnant for the first time at the age of 27 or older. In the same study no relationship was found between ever having been pregnant and cancer of the biliary tract. However, a correlation was observed between the number of pregnancies and the risk of cancer. The higher levels of estrogen and progesterone during pregnancy lead to increased secretion of cholesterol into the bile [21]. Pregnancy also impairs contractility of GB, which not only facilitates the formation of gallstones, but also leads to long-term exposure of the mucosa to other components of bile with potential carcinogenic properties.

\section{Xanthogranulomatous cholecystitis}

Xanthogranulomatous cholecystitis (XGC) is an uncommon inflammatory disease of GB. It is characterized by the presence of multiple intramural nodules, proliferative fibrosis and infiltration of macrophages and foamy cells [22]. XGC is associated with gallbladder carcinoma in $8.5 \%$ to $30.5 \%$ cases [23]. Pre-operative differentiation of xanthogranulomatous cholecystitis from GBC both on the basis of the clinical picture and radiological features is a great diagnostic challenge. It is also important that both of these diseases often coexist in one patient. The destructive effect of GBC on the gallbladder wall may cause bile to enter the GB stroma and result in xanthogranulomatous cholecystitis. A relationship with gallbladder carcinoma is still uncertain [24].

\section{Lipid peroxidation products}

The gall bladder is a reservoir of waste products of the liver. It seems likely that when combined with cholelithiasis and cholestasis, exposure to harmful metabolic products may induce GBC. 4-hydroxynonenals (HNE) is a major product of liver microsomal lipid peroxidation and is transported with bile from the liver to GB. Of all the lipid peroxidation products, it is the most significantly mutagenic and has high neoplastic potential [25]. Long-term persistence of high HNE concentrations in bile may be involved in development of GBC. The level of HNE in the bile of cancer patients was notably higher [26].

Attention is drawn to the higher incidence of GBC among workers in the rubber, automobile, and metal fabricating industries. The commonly used carbon tetra chloride is accumulated in the liver and causes initiation and stimulation of microsomal lipid peroxidation [27]. Peroxidation products such as HNE accumulate in the gallbladder and may contribute to tumour formation. 


\section{Aflatoxins}

Aflatoxins produced by Aspergillus fungi contaminate foods prepared from infected cereals, oilseeds, nuts, spices, milk, and meats with fungus and that are kept under favourable conditions. Aflatoxins are one of the risk factors for the development of hepatocellular carcinoma (HCC). It seems that aflatoxins are also involved in GBC development. Chronic exposure of the gallbladder mucosa epithelium to carcinogenic metabolites of aflatoxins accumulated in bile may cause dysplasia and, consequently, GBC [28]. In one study [28] high consumption of red chilli pepper has been shown to be a risk factor for gallbladder cancer. This was considered a consequence of aflatoxins contamination of peppers, and long-term exposure to aflatoxins may be associated with the development of GBC.

\section{Conclusions}

Gallbladder cancer was first described nearly 250 years ago. Despite many studies and intensive efforts of scientists, the etiopathogenesis of GBC remains unclear. This cancer appears to be a multifactorial disease. The main risk factors for GBC are: cholelithiasis and porcelain gallbladder as a result of chronic cholecystitis. Anomalous junction of the pancreaticobiliary ductal system and chronic infection of the gallbladder with Helicobacter pylori have been also suggested as a risk factor for GBC. Attention is also drawn to the fact that elderly women most often develop GBC, which suggests a relationship between the incidence of this cancer and age and gender. According to recent studies, aflatoxins and lipid peroxidation products may also play a role in the formation of GBC. In order to thoroughly carcinogenesis of GBC more studies concerning the risk factors have to be conducted. A deeper understanding of the biological mechanisms responsible for development of GBC may allow to establish reliable biomarkers as well as invent new preventive strategies and more effective treatment.

\section{References}

[1] I. Roa, J.C. Araya, M. Villaseca, et al.; Preneoplastic lesions and gallbladder cancer: an estimate of the period required for progression; Gastroenterology, 111 (1996), pp. 232-236

[2] D.L. Bartlett; Gallbladder cancer; Semin Surg Oncol, 19 (2000), pp. 145-155

[3] I. Roa, X. de Aretxabala, J.C. Araya, et al.; Preneoplastic lesion in gallbladder cancer; J Surg Oncol, 93 (2006), pp. 615-623

[4] Nowotwory pęcherzyka żółciowego i przewodów żółciowych zewnątrzwątrobowych; http://onkologia.org.pl

[5] Everson GT, McKinley C, Kern F. Mechanisms of gallstone formation in women. Effects of exogenous estrogen (Premarin) and dietary cholesterol on hepatic lipid metabolism. J Clin Invest. 1991;87:237-246.

[6] M. Carriaga, D.E. Henson; Liver, gallbladder, extrahepatic bile ducts, and pancreas; Cancer, 75 (1995), pp. $171-190$

[7] B. Strom, R.D. Soloway, J.L. Rios-Dalenz, et al.; Risk factors for gallbladder cancer: An international collaborative case control study Cancer, 76 (1995), pp. 1747-1756

[8] A.K. Diehl; Epidemiology of gallbladder cancer: a synthesis of recent data J Natl Cancer Int, 65 (1980), pp. 1209-1214

[9] A.K. Diehl; Gallstone size and the risk of gallbladder cancer JAMA, 250 (1983), pp. 2323-2326

[10] S. Towfigh, D.W. McFadden, G.R. Cortina, et al.; Porcelain gallbladder is not associated with gallbladder carcinoma,; Am Surg, 67 (2001), pp. 7-10

[11] A.E. Stephen, D.L. Berger; Carcinoma in the porcelain gallbladder: a relationship revisited; Surgery, 129 (2001), pp. 699-703 
[12] M.C. Aldridge, H. Bismuth; Gallbladder cancer: the polyp cancer sequence; Br J Surg, 77 (1990), pp. 363 364

[13] Tanaka K, Ikoma A, Hamada N, Nishida S, Kadono J, Taira A. Biliary tract cancer accompanied by anomalous junction of pancreaticobiliary ductal system in adults. Am J Surg. 1998;175:218-220.

[14] Park M, Song DY, Je Y, Lee JE. Body mass index and biliary tract disease: a systematic review and metaanalysis of prospective studies. Prev Med. 2014;65:13-22

[15] R.C. van Kruijsdijk, E. van der Wall, F.L. Visseren; Obesity and cancer: the role of dysfunctional adipose tissue; Cancer Epidemiol. Biomarkers Prev., 18 (10) (2009), pp. 2569-2578

[16] Jackson SS, Van Dyke AL, Zhu B, et al. Anthropometric Risk Factors for Cancers of the Biliary Tract in the Biliary Tract Cancers Pooling Project. Cancer Res 2019;79:3973-82.

[17] Solnick JV, Schauer BD. Emergence of diverse Helicobacter species in the pathogenesis of gastric and enterohepatic diseases. Clin. Microbiol. Rev. 2001; 14: 59-97.

[18] Hughes NR, Bhathal PS. Adenocarcinoma of gallbladder: an immunohistochemical profile and comparison with cholangiocarcinoma. J Clin Pathol. 2013;66(3):212-7.

[19] Murphy G, Michel A, Taylor PR, Albanes D, Weinstein SJ, Virtamo J, et al. Association of seropositivity to Helicobacter species and biliary tract cancer in the ATBC study. Hepatology. 2014;60(6):1963-71.

[20] Moerman CJ, Berns MP, Bueno de Mesquita HB, Runia S. Reproductive history and cancer of the biliary tract in women. Int J Cancer. 1994;57:146-153.

[21] Carey, M.C., Cahalane, M.J., Whither biliary sludge. Gastroenterology, 95,508-523 (1988).

[22] Rammohan A, Cherukuri SD, Sathyanesan J, Palaniappan R, Govindan M. Xanthogranulomatous cholecystitis masquerading as gallbladder cancer: can it be diagnosed preoperatively? Gastroenterol Res Pract. 2014;2014:253645.

[23] Krishnani N, Shukla S, Jain M, Pandey R, Gupta RK. Fine needle aspiration cytology in xanthogranulomatous cholecystitis, gallbladder adenocarcinoma and coexistent lesions. Acta Cytol. 2000;44:508-514.

[24] Dixit VK, Prakash A, Gupta A et-al. Xanthogranulomatous cholecystitis. Dig. Dis. Sci. 1998;43 (5): $940-2$.

[25] Esterbauer H, Zollner H, Schaur RJ: Hydroxyalkenals: Cytotoxic products of lipid peroxidation. IS1 Atlas Sci Biochem 1:311-315, 1988.

[26] Shukla VK, Shukla PK, Pandey M, Rao BR, Roy SK. Lipid peroxidation product in bile from patients with carcinoma of the gallbladder: a preliminary study. J Surg Oncol. 1994;56:258-262.

[27] Slater TF, Sawyer BC: The stimulatory effects of carbon tetra chloride on peroxidative reaction in rat liver fractions in vitro. Interaction sites in the endoplasmic reticulum. Biochem $\mathrm{J}$ 123:

[28] Tsuchiya Y, Terao M, Okano K, et al. Mutagenicity and mutagens of the red chilli pepper as gallbladder cancer risk factor in Chilean women. Asian Pac J Cancer Prev 2011;12:471-6. 